\title{
Non-thermal line-broadening in solar prominences
}

\author{
G. Stellmacher ${ }^{1}$ and E. Wiehr ${ }^{2}$ \\ ${ }^{1}$ Institute d'Astrophysique (IAP), 98bis bd d'Arago, 75014 Paris, France \\ 2 Institut für Astrophysik der Universität, Friedrich-Hund-Platz 1, 37077 Göttingen, Germany \\ e-mail: ewiehr@astrophysik.uni-goettingen.de
}

Received 2 October 2013 / Accepted 15 July 2015

\begin{abstract}
Aims. We show that the line broadening in quiescent solar prominences is mainly due to non-thermal velocities.

Methods. We have simultaneously observed a wide range of optically thin lines in quiescent prominences, selected for bright and narrow $\mathrm{Mg}$ b emission without line satellites from macro-shifts.

Results. We find a ratio of reduced widths, $\Delta \lambda_{\mathrm{D}} / \lambda_{0}$, of $\mathrm{H} \gamma$ and $\mathrm{H} \delta$ of $1.05 \pm 0.03$, which can hardly be attributed to saturation, since both are optically thin for the prominences observed: $\tau_{\gamma} \leq 0.3, \tau_{\delta} \leq 0.15$. We confirm the ratio of reduced widths of He 4772 (triplet) and $\mathrm{He} 5015$ (singlet) of $1.1 \pm 0.05$ at higher significance and detect a width ratio of $\mathrm{Mg} \mathrm{b}_{2}$ and $\mathrm{Mg} 4571$ (both from the triplet system) of $1.3 \pm 0.1$

Conclusions. The discrepant widths of lines from different atoms, and even from the same atom, cannot be represented by a unique pair $\left[T_{\text {kin }} ; V_{\text {nth }}\right]$. Values of $T_{\text {kin }}$ deduced from observed line radiances using models indicate low temperatures down to $T_{\text {kin }} \approx 5000 \mathrm{~K}$. Non-thermal velocities, related to different physical states of the respective emitting prominence region, seem to be the most important line broadening mechanism.
\end{abstract}

Key words. Sun: filaments, prominences

\section{Introduction}

An essential parameter for the description of a prominence plasma is its temperature, usually derived from the widths of optically thin lines. Widths can be observed with high accuracy and are independent of the calibration in absolute units, unlike an analysis using line radiance data. The large spectral resolution of ground-based spectrographs (typically $\lambda / \Delta \lambda \approx 4 \times 10^{5}$ ) assures a negligible influence of the instrumental profile on the narrow prominence emission lines. In contrast, existing spaceborn spectrographs (as e.g. SUMER on-board SOHO), designed for broad coronal emissions, strongly affect the widths of prominence lines (cf. Stellmacher et al. 2003).

Prominence emission lines are generally assumed to be broadened by Doppler motions resulting from thermal $\left(V_{\mathrm{th}}=\right.$ $\left.\sqrt{2 R T_{\mathrm{kin}} / \mu}\right)$ and non-thermal $\left(V_{\mathrm{nth}}\right)$ velocities. The separation of both requires (at least two) lines from atoms of different mass $\mu$ originating in the same prominence volume. Clearly, it is advantageous to use as broad a range of lines as possible. However, apart from the first Balmer and the Ca II lines, prominence emissions in the visible spectrum are faint and thus difficult to observe at sufficiently high spectral resolution. Extended ground-based observations of faint metallic lines at high spectral resolution have been almost exclusively published by Landman and collaborators (e.g. Landman 1985).

Comparing the Doppler widths of $\mathrm{NaD}_{1,2}$ with those of $\mathrm{He} \mathrm{D}_{3}$ and Ca II 8498, Landman (1981) found that "the assumption of a common emission region for these lines appears to have limited validity". Recently, Ramelli et al. (2012) and Wiehr et al. (2013) resumed such observations and found that "cool" emissions (e.g. of $\mathrm{NaD}$ or $\mathrm{Mg}$ ) are accompanied by rather broad

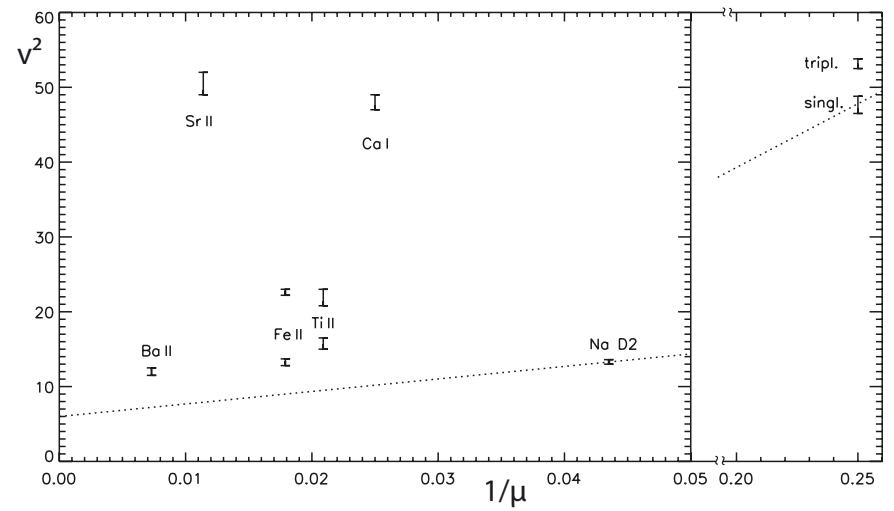

Fig. 1. Observed line widths $V^{2}=\left(c \cdot \Delta \lambda_{\mathrm{D}} / \lambda_{0}\right)^{2}\left[\left(\mathrm{~km} \mathrm{~s}^{-1}\right)^{2}\right]$ versus inverse atomic mass $0 \leq 1 / \mu \leq 0.05$ of metallic lines and $0.20 \leq 1 / \mu \leq$ 0.26 for helium singl. and tripl. (from Ramelli et al. 2012; listed in their Table 3, here with error bars). The dotted line connects $\mathrm{Na}_{2}$ and He singlet with apparent $T_{\text {kin }}=9950 \mathrm{~K}$ and $V_{\text {nth }}=2.5 \mathrm{~km} \mathrm{~s}^{-1}$ but does not fit any width of the other emission lines. The range $0.05 \leq 1 / \mu \leq$ 0.20 is omitted.

$\mathrm{H} \gamma$ lines of widths corresponding to $T_{\text {kin }}>10000 \mathrm{~K}$, and conclude that observed Doppler widths cannot simply be described by a unique pair $\left[T_{\text {kin }} ; V_{\text {nth }}\right]$. In Fig. 1 we summarize the data by Ramelli et al. (2012; listed in their Table 3, but here with error bars) as a diagram of $\left(c \cdot \Delta \lambda_{\mathrm{D}} / \lambda_{0}\right)^{2}$ versus the inverse atomic mass, showing the large deviation from a linear relation expected for a unique $\left[T_{\text {kin }} ; V_{\text {nth }}\right]$. These observations were taken consecutively and might thus be influenced by time evolution or drifts of the prominence over the spectrograph slit. 
Table 1. Date and heliographic position of the prominences.

\begin{tabular}{|c|c|c|c|}
\hline \multicolumn{4}{|c|}{ THEMIS } \\
\hline 1 & June, 06 & 2013 & west limb, $25^{\circ} \mathrm{N}$ \\
\hline 2 & June, 07 & & west limb, $21^{\circ} \mathrm{N}$ \\
\hline 3 & June, 10 & & west limb, $22^{\circ} \mathrm{N}$ \\
\hline 4 & June, 10 & & west limb, $33^{\circ} \mathrm{S}$ \\
\hline 5 & June, 11 & & west limb, $22^{\circ} \mathrm{N}$ \\
\hline 6 & June, 11 & & east limb, $20^{\circ} \mathrm{S}$ \\
\hline 7 & June, 12 & & west limb, $38^{\circ} \mathrm{N}$ \\
\hline 8 & June, 12 & & west limb, $25^{\circ} \mathrm{N}$ \\
\hline 9 & June, 12 & & west limb, $68^{\circ} \mathrm{S}$ \\
\hline \multicolumn{4}{|c|}{ IRSOL } \\
\hline 10 & Oct., 09 & 2013 & west limb, $20^{\circ} \mathrm{S}$ \\
\hline 11 & Oct., 11 & & west limb, $23^{\circ} \mathrm{N}$ \\
\hline 12 & Oct., 16 & & west limb, $05^{\circ} \mathrm{N}$ \\
\hline 13 & Oct., 16 & & east limb, $30^{\circ} \mathrm{N}$ \\
\hline 14 & Oct., 17 & & west limb, $38^{\circ} \mathrm{S}$ \\
\hline 15 & June, 30 & 2014 & west limb, $13^{\circ} \mathrm{S}$ \\
\hline
\end{tabular}

\section{Observations}

\subsection{THEMIS data}

We obtained simultaneous spectra of the prominence emissions $\mathrm{H} \gamma, \mathrm{He} 5015$ (singlet) with Fe II 5018, He 4472 (triplet) with Ti II 4468, He II 4686 and $\mathrm{Mg} \mathrm{b}_{1}$ (replacing the former $\mathrm{Mg}_{2}$ line for technical reason) with five CCD cameras and $10 \mathrm{~s}$ exposure time with the $90 \mathrm{~cm}$ THEMIS telescope on Tenerife in June 2013. As for the former observations at the Locarno observatory, IRSOL (cf. Ramelli et al. 2012), we selected quiescent prominences with bright but narrow Mgb emissions without marked macro shifts, thus assuring a "spectral photon concentration". The positions of the observed prominences are listed in Table 1.

For a sufficiently high signal-to-noise ratio, we chose a slit with of $1.5 \operatorname{arcsec}$ corresponding to $1000 \mathrm{~km}$ on the sun. The slit was oriented in the direction of atmospheric refraction, i.e. perpendicular to the horizon. Wavelength dependent refraction in the earth's atmosphere $(\leq 0.2$ arcsec through our spectral range $4340 \AA<\lambda<5152 \AA$ ) then moves the prominence structures along the slit, thus preserving them in the different spectra, where they can readily be considered by suitable spatial shifts of the respective scans.

We took spectra of the close (emission free) prominence neighbourhood immediately after each exposure for a determination of stray light, mainly originating from the telescope mirrors (rather than the earth's atmosphere; cf. Stellmacher \& Wiehr 1970). Additional stray light from the spectrograph mirrors was minimized by excluding light from the solar disk.

For a determination of absolute spectral radiance values, we took spectra of the disk centre with an integration time of $1 \mathrm{~s}$. Ramelli et al. (2012) describe the reduction procedure. We integrated the line profiles over 15 pixels in the spatial direction, corresponding to $3.4 \mathrm{arcsec}$, i.e. $2500 \mathrm{~km}$ on the sun. The final emission line profiles were fitted by Gaussians.

\subsection{Further IRSOL data}

Since THEMIS restricts the combination of lines to $\Delta \lambda<$ $800 \AA$ and to appropriate grating orders and since the THEMIS data confirmed the former sequential results (see Sect. 3), we took further spectra at IRSOL that allowed us to extend existing observations to more than one line from the same atom: Additionally to the lines taken with THEMIS, we observed $\mathrm{H} \delta$

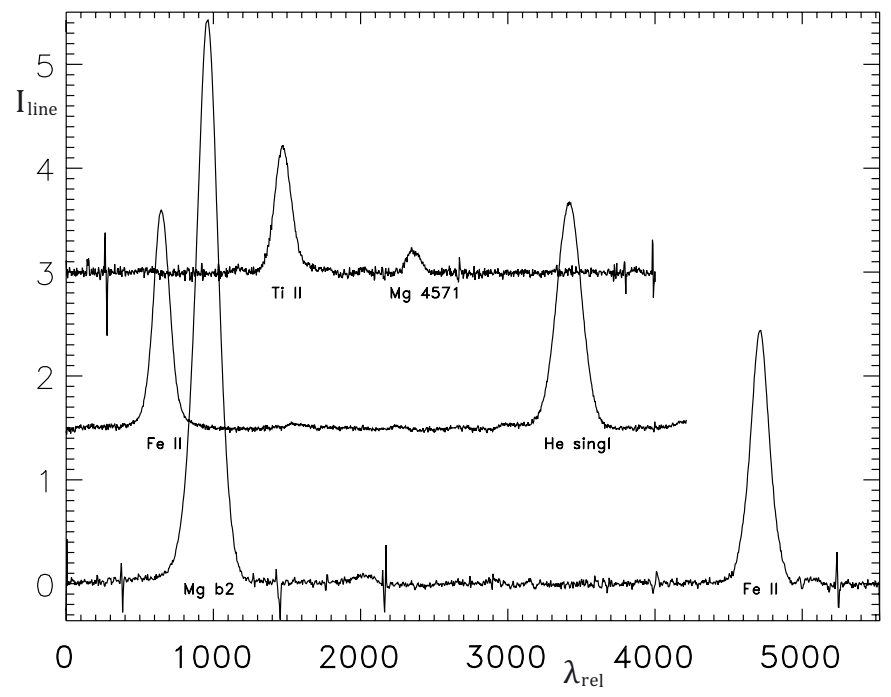

Fig. 2. Spectral line radiance, $I_{\text {line }}\left[\mathrm{erg} /\left(\mathrm{s} \mathrm{cm}^{2}\right.\right.$ ster $\left.\mathrm{m} \AA\right]$, of Fe II 5168 with $\mathrm{Mg} \mathrm{b}_{2} 5172$ (lower), He 5015 (singl.) with Fe II 5018 (middle), ordinate shifted by $+1.5 \mathrm{erg} /\left(\mathrm{s} \mathrm{cm}^{2}\right.$ ster $\left.\mathrm{m} \AA\right)$; and $\mathrm{Mg} 4571$ with Ti II $4572 \AA$ (upper curve), shifted by $+3.0 \mathrm{erg} /\left(\mathrm{s} \mathrm{cm}^{2}\right.$ ster $\mathrm{m} \AA$ ), observed in prominence-15 (cf. Table 1). The abscissa gives relative

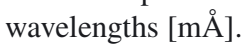

for comparison with $\mathrm{H} \gamma$ (in October 2013) and $\mathrm{Mg} 4571$ for comparison with Mg b2 (in June 2014, cf. Table 1).

\subsection{Accuracy of the line widths}

The errors of the emission line widths arise (a) from the apparent continuum level between the emission lines and (b) from the quality of the fit. The spurious rest of the stray light (a) depends on the correct subtraction of a spectrum from the (emission free) vicinity of the prominence. We determine zero as mean (count) level between the emissions. For the noisiest profile, $\mathrm{Mg} 4571$ (see Fig. 2), the rms of this zero level amounts to typically $1 \%$ of the counts in the emission maximum. This leads to an error of the $\mathrm{Mg} 4571$ widths of $\pm 7.5 \%$. The other emission line widths are of much higher accuracy (near 1\%). For the strong Balmer emissions the zero-level uncertainty yields a width error well below $1 \%$.

In contrast with the accuracy of the zero-level (a), that of the fit (b) is one order of magnitude higher, since the optically thin profiles appear to be well-defined Gaussians. Figure 2 gives three spectral scans, each covering two neighbouring prominence emissions, showing the high signal-to-noise ratio.

Besides $\mathrm{Mg}$ 4571, the lowest signal-to-noise ratio is found for the profile of He II $4686 \AA$, which is only measurable in extremely bright (and rare) prominences. Figure 3 shows that even this line profile can be fitted to an accuracy of $\leq 10 \%$.

\section{Results}

The results from the simultaneous THEMIS observations fully confirm those from IRSOL in 2011 and 2012, demonstrating that the widths discrepancies found from sequential data at IRSOL are not an artifact of short-time evolution or drifts of the prominences over the spectrograph slit. Comparing the observed widths of the various emissions from different atoms, we again find with THEMIS that none of the sets of lines observed 


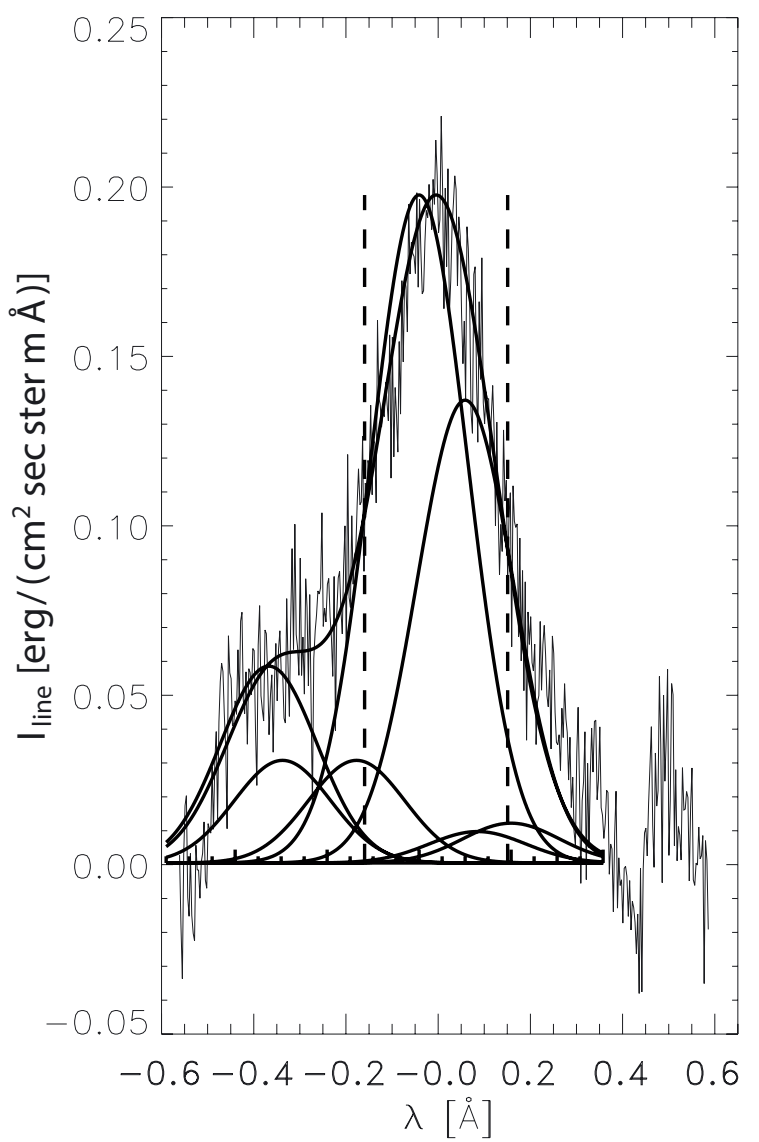

Fig. 3. He II $4686 \AA$ emission, observed in prominence-15. It is fitted by a profile composed of the seven fine-structure components (cf. Ramelli et al. 2012) each with $F W H M_{\text {int }}=260 \mathrm{~m} \AA$, yielding a superposed profile with $F W H M_{\text {tot }}=320 \mathrm{~m} \AA$ as observed.

in a given prominence can be represented by a unique pair of $\left[T_{\text {kin }} ; V_{\text {nth }}\right]$ in the well-known relation for Doppler broadening, i.e.

$$
\left(V_{\mathrm{D}}\right)^{2}=\left(c \cdot \Delta \lambda_{\mathrm{D}} / \lambda_{0}\right)^{2}=2 R T_{\text {kin }} / \mu+V_{\text {nth }}^{2}
$$

where $\mu$ is the atomic mass and $V_{\text {nth }}$ the non-thermal line broadening parameter. We establish the ambiguity of $T_{\text {kin }}$ and $V_{\text {nth }}$ shown in Fig. 1. This finding is even more impressively seen in Fig. 4, where we attribute to each reduced width, $\Delta \lambda_{\mathrm{D}} / \lambda_{0}$, a possible combination $\left[T_{\text {kin }} ; V_{\text {nth }}\right]$, i.e.

$$
V_{\mathrm{nth}}=V_{\mathrm{D}}^{\mathrm{obs}}-\sqrt{2 R T_{\mathrm{kin}} / \mu}
$$

Figure 4 shows that line widths of atoms with high mass, $\mu$, are largely insensitive to $T_{\text {kin }}$, in contrast to the Balmer lines. The variety of curves excludes a unique $\left[T_{\text {kin }} ; V_{\text {nth }}\right]$ pair fitting the various emission line widths. The apparent coincidence of $\mathrm{H} \gamma$, He singlet and $\mathrm{Mg} 4571$ is rather fortuitous and does not occur in similar diagrams for other prominences. Evidently, combinations of lines from atoms of different mass, $\mu$, required to separate thermal and non-thermal broadening, give contradicting pairs of $\left[T_{\text {kin }} ; V_{\text {nth }}\right]$, which cannot unambiguously relate to corresponding common emission regions within the respective prominence.

\subsection{Balmer lines}

The quiescent prominences selected for bright but narrow $\mathrm{Mgb}$ emission persistently show broad $\mathrm{H} \gamma$ emissions with

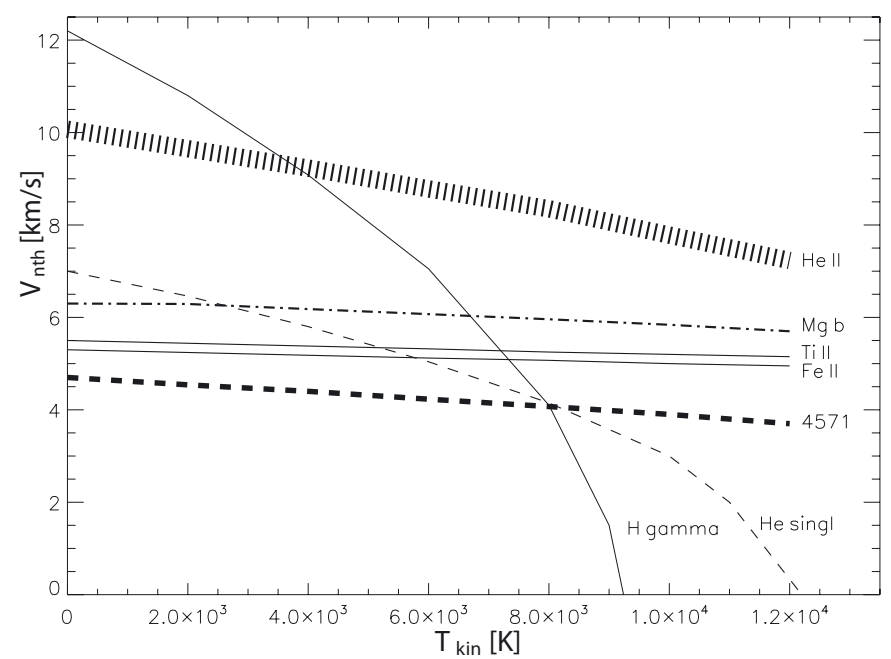

Fig. 4. Relation of $V_{\text {nth }}$ and $T_{\text {kin }}$ for each reduced line width, $\Delta \lambda_{\mathrm{D}}^{\text {obs }} / \lambda_{0}$, observed in prominence-15. The thickness of each curve represents the error bar of each observed $\Delta \lambda_{\mathrm{D}} / \lambda_{0}$.

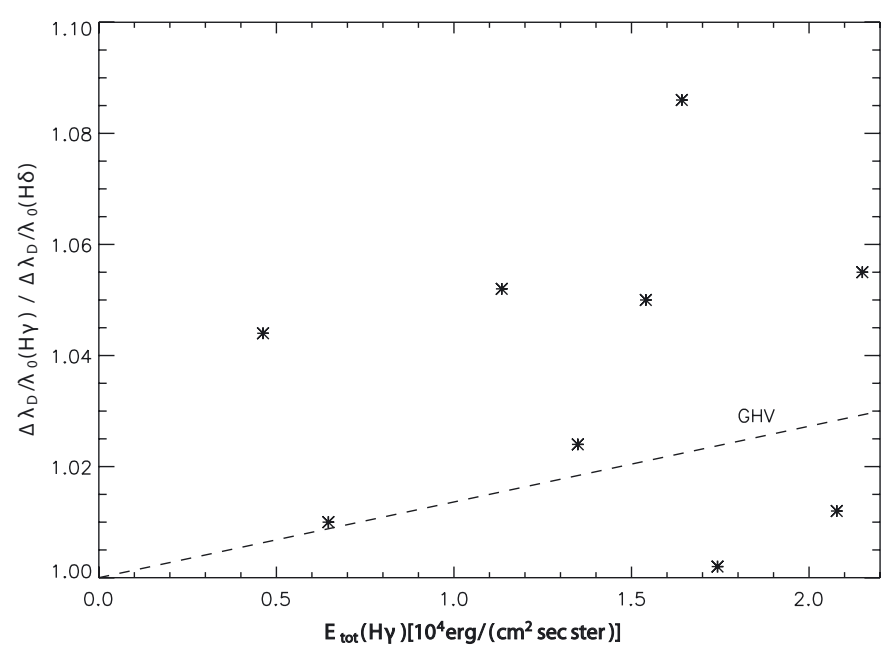

Fig. 5. Ratio of observed reduced Doppler widths $\Delta \lambda_{\mathrm{D}} / \lambda_{0}$ of $\mathrm{H} \gamma$ and $\mathrm{H} \delta$ versus the total emission ("line radiance") of $\mathrm{H} \gamma$. The error bars are smaller than the symbols. Dashes give a mean of the three models with $T_{\text {kin }}=4300,6000$ and $8000 \mathrm{~K}$ (all $V_{\text {nth }}=5 \mathrm{~km} \mathrm{~s}^{-1}$ ) by Gouttebroze et al. (1993).

reduced widths $4.1 \times 10^{-5} \leq \Delta \lambda_{\mathrm{D}} / \lambda_{0} \leq 5.2 \times 10^{-5}$. For purely thermal broadening (i.e. $V_{\text {nth }}=0 \mathrm{~km} \mathrm{~s}^{-1}$ ), this would correspond to $9200 \leq T_{\text {kin }} \leq 14800 \mathrm{~K}$.

To get more information about the broadening of Balmer lines, we observed $\mathrm{H} \delta$ together with $\mathrm{H} \gamma$ in Oct. 2013 at IRSOL and confirm the large widths of these two optically thin lines. The analysis shows that the ratio of the reduced Doppler widths of $\mathrm{H} \gamma$ and $\mathrm{H} \delta$ is larger than 1.0 and does not systematically vary with the $\mathrm{H} \gamma$ radiance (Fig. 5).

The isothermal model calculations by Gouttebroze et al. (1993, hereafter referred to as GHV) yield a width ratio of $\mathrm{H} \gamma / \mathrm{H} \delta$ which increases to 1.03 through our observed radiance $E \gamma \leq 2.2 \times 10^{4} \mathrm{erg} /\left(\mathrm{s} \mathrm{cm}^{2}\right.$ ster $)$. For this range, the models give a total optical thickness in the respective emission line centres of $\tau_{\delta} \leq 0.15, \tau_{\gamma} \leq 0.3,\left(\tau_{\alpha} \leq 8\right)$, and predict an increase of the mean-width excess $\mathrm{H} \gamma / \mathrm{H} \delta$ with brightness (dashed line in Fig. 5) independent of the assumed model temperature $\left(T_{\text {kin }}=4300\right.$, 6000 and $8000 \mathrm{~K}$; all $V_{\text {nth }}=5 \mathrm{~km} \mathrm{~s}^{-1}$ ). Our observed width ratios 


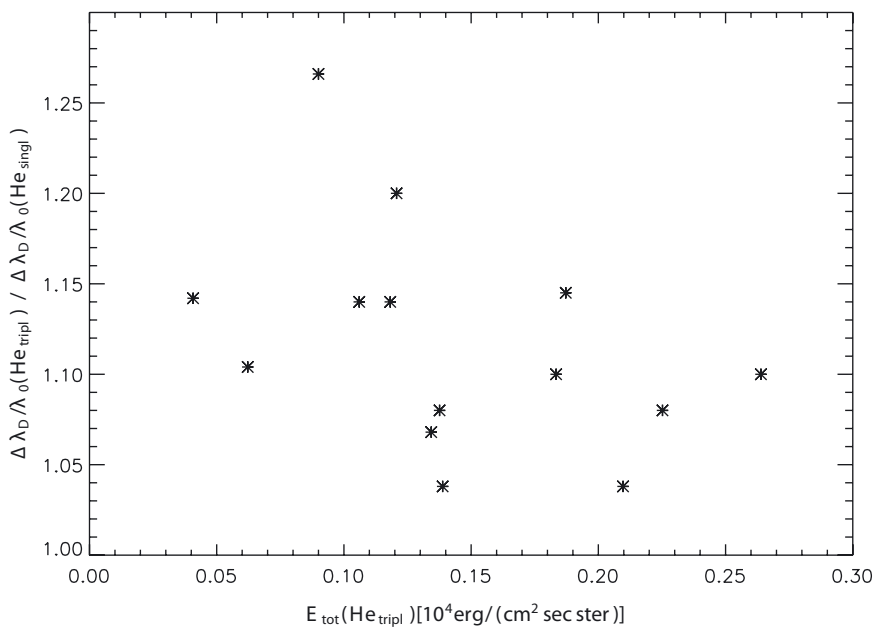

Fig. 6. Ratio of observed reduced Doppler widths $\Delta \lambda_{D} / \lambda_{0}$ of He I 4472 (triplet) and HeI5015 (singlet) versus the total emission ("line radiance") of $\mathrm{He}$ I (triplet). The 1\% accuracy (cf. Sect. 2.3) yields error bars of both, the ratio and the triplet radiance values slightly larger than the figure symbols.

deviate from these model calculations and cannot be explained by saturation effects.

\subsection{Helium lines}

The simultaneous observations with THEMIS confirm those, which have been taken consecutively at IRSOL. For the reduced widths $\Delta \lambda_{D} / \lambda_{0}$, the mean ratio of He II $4686 \AA$ and He I $4472 \AA$ is found to be $1.45 \pm 0.15$ (former value 1.5) and that of He I $4472 \AA$ (triplet) and He I $5015 \AA$ (singlet) remains $1.1 \pm 0.05$ (at higher significance). There is no marked dependence on the brightness of the respective prominence, e.g. on the line radiance of He I triplet (Fig. 6). Thus the variety of the width ratios of He triplet and He singlet cannot be explained by saturation effects (just like the two Balmer lines). Ramelli et al. (2012) suggest that the line from the triplet system reflects a hotter origin than the singlet line, and is populated via ionization and recombination.

For the line radiance, we obtain a slightly smaller ratio of He I 4472 (triplet) and He II 4686 of 45 (formerly 50), whereas that of He (triplet) and He (singlet) of 9.4 (Wiehr et al. 2013) remains unchanged.

\subsection{Magnesium lines}

The faint triplet-to-singlet inter-combination line $\mathrm{Mg} 4571 \AA$, observed by Sotirovski (1965) during eclipse, can only be measured in very bright prominences at high spectral resolution. Ramelli et al. (2012) find a small reduced width of $\Delta \lambda_{\mathrm{D}} / \lambda_{0}(4571)=0.9 \times 10^{-5}$. We verified this at Locarno in Oct. 2013 and again find $\Delta \lambda_{\mathrm{D}} / \lambda_{0}=1.0 \times 10^{-5}$. On June, 30, 2014 we observed an exceptionally bright prominence at W/13S $\left(E_{\mathrm{tot}}^{\alpha} \approx 4.4 \times 10^{5} \mathrm{erg} /\left(\mathrm{s} \mathrm{cm}^{2} \mathrm{ster}\right)\right.$, i.e. $\tau_{\mathrm{alpha}} \approx 20 \mathrm{in}$ the GHV tables), where $\mathrm{Mg} 4571$ had the high line radiance of $50 \mathrm{erg} /\left(\mathrm{s} \mathrm{cm}^{2}\right.$ ster $)$. We found that the $\mathrm{Mg} \mathrm{b}_{2}$ line is $1.3 \pm 0.1$ times broader than $\mathrm{Mg}$ 4571. This cannot be due to saturation, since our radiance value of $E_{\mathrm{tot}}\left(\mathrm{Mg} \mathrm{b}_{2}\right)=1750 \mathrm{erg} /\left(\mathrm{s} \mathrm{cm}^{2} \mathrm{ster}\right)$ is much smaller than the $6700 \mathrm{erg} /\left(\mathrm{s} \mathrm{cm}^{2} \mathrm{ster}\right)$ observed by Landman (1985), for which he estimates $\tau_{0}\left(\mathrm{Mg} \mathrm{b}_{2}\right) \approx 0.7$; besides, our $\mathrm{Mg} \mathrm{b}_{2}$ profile is perfectly Gaussian.
The width excess of $\mathrm{Mg} \mathrm{b}_{2}$ over $\mathrm{Mg} 4571$ cannot be explained in the same way as that of He triplet over He singlet, since both upper $\mathrm{Mg}$ levels belong to the triplet system. The upper level of $\mathrm{Mg} 4571\left(3^{3} \mathrm{P}_{1}\right)$ is populated by the $\mathrm{Mg} \mathrm{b}$ transition; the large width excess of $\mathrm{Mg} \mathrm{b} \mathrm{b}_{2}$ over $\mathrm{Mg} 4571$ is thus hard to understand. On the other hand, the $3{ }^{3} \mathrm{P}_{1}$ level is meta-stable and can be populated from the lowest $\mathrm{Mg}$ level $\left(1^{1} \mathrm{P}_{0}\right.$; singlet) by collisions. In that case, the narrow $\mathrm{Mg} 4571$ width might indicate that these collisions only occur in prominence regions with small line broadening.

\section{Discussion}

We have analysed a comprehensive set of faint metallic lines in quiescent prominences, which hitherto have rarely been observed at high spectral resolution. A sufficiently high signal-tonoise ratio requires prominences that show narrow, but bright emissions largely free from macro-shifts (assuring a "spectral photon concentration"). From our former search of measurable He II $4686 \AA$ emission, we know that suitable prominences are massive, poorly structured and show pronounced $\mathrm{NaD}$ emission. The simultaneous appearance of "hot" He II and "cool" $\mathrm{Na}$ I lines already indicates emissions from different prominence volumes. Our various observations with different telescopes, spectrographs and line combinations indeed show that the line widths do not allow one to unambiguously separate thermal and non-thermal line broadening. A determination of kinetic temperatures in quiescent prominences from widths analyses thus remains highly uncertain. Given the limits and uncertainties (e.g. Figs. 1 and 4), we are not surprised by the large ranges deduced from widths observed in "cool parts of prominences": $6000<T_{\text {kin }}<15000 \mathrm{~K}$ (Jejcic et al. 2014) and even $4000<$ $T_{\text {kin }}<20000 \mathrm{~K}$ (Park et al. 2013).

\section{1. $T_{\text {kin }}$ from line radiances}

Line radiances offer another way to get information about physical conditions in prominences. A remarkably tight relation of $\mathrm{H} \alpha$ and $\mathrm{H} \beta$ emissions over a large brightness range up to $E^{\beta}=1.5 \times 10^{5} \mathrm{erg} /\left(\mathrm{s} \mathrm{cm}^{2}\right.$ ster $)$ is found when superposing observations at Tenerife with the VTT-spectrograph (Fig. 2 in Stellmacher \& Wiehr 1994), from 2D imaging at the VTT (Fig. 4 in Stellmacher \& Wiehr 2000), and from spectra with THEMIS (Fig. 3a in Stellmacher \& Wiehr 2005). That relation (shown in Wiehr \& Stellmacher 2015) is at best represented by the GHV models with $T_{\text {kin }}=4300 \mathrm{~K}$ and $T_{\text {kin }}=6000 \mathrm{~K}$ (both $V_{\text {nth }}=5 \mathrm{~km} \mathrm{~s}^{-1}$ ) Gunár et al. (2012) use 2D multi-thread models with prominence-corona transition region, PCTR, to reproduce observed $\mathrm{H} \alpha$ radiances and widths, and deduce similar low temperatures down to $T_{\text {kin }} \approx 5000 \mathrm{~K}$.

Since none of our various $V_{\mathrm{D}}^{2}$ versus $1 / \mu$ diagrams (e.g. Fig. 1) gives gradients corresponding to such low $T_{\text {kin }}$, the lines will be broadened mainly by non-thermal velocities. Adopting the above $5000 \mathrm{~K}$, the observed $\mathrm{H} \gamma$ widths $4.1 \leq \Delta \lambda_{\mathrm{D}} / \lambda_{0} \leq 5.2 \times$ $10^{-5}$ would give $8.3 \leq V_{\text {nth }} \leq 12.7 \mathrm{~km} \mathrm{~s}^{-1}$ and the $5 \%$ smaller $\mathrm{H} \delta$ widths $7.9 \leq V_{\text {nth }} \leq 12.1 \mathrm{~km} \mathrm{~s}^{-1}$; assuming $T=5000 \mathrm{~K}$ for the observed Mg lines, the $\mathrm{Mg} \mathrm{b}_{2}$ widths $1.4 \leq \Delta \lambda_{\mathrm{D}} / \lambda_{0} \leq 1.7 \times 10^{-5}$ would yield $3.8 \leq V_{\text {nth }} \leq 4.8 \mathrm{~km} \mathrm{~s}^{-1}$, and the 1.3-times narrower $\operatorname{Mg} 4571$ line $2.9 \leq V_{\text {nth }} \leq 3.7 \mathrm{~km} \mathrm{~s}^{-1}$.

\subsection{Non-thermal velocities}

This strong and variable influence of $V_{\text {nth }}$ on the prominence emission line widths is also supported by the spatial variation 


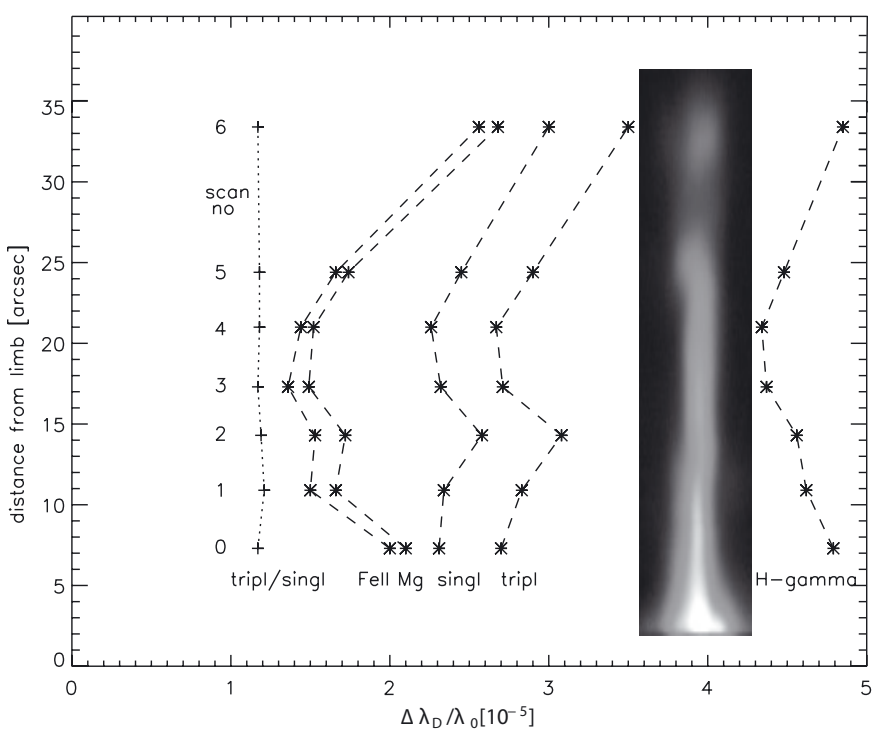

Fig. 7. Spatial variation (i.e. along the slit) of reduced Doppler widths $\Delta \lambda_{\mathrm{D}} / \lambda_{0}$ of $\mathrm{H} \gamma, \mathrm{He} \mathrm{I} 4472$ (triplet), He I 5015 (singlet), $\mathrm{Mg} \mathrm{b}_{2} 5172$, and Fe II 5018; also shown the (nearly constant) width ratio of He (tripl) and $\mathrm{He}$ (singl). The prominence No. 1 by chance extends along the direction of refraction, which necessarily is the slit orientation, cf. Sect. 2.1. Inserted is the $\mathrm{Mg} \mathrm{b}_{2}$ emission spectrum; $\Delta \lambda=0.8 \AA$.

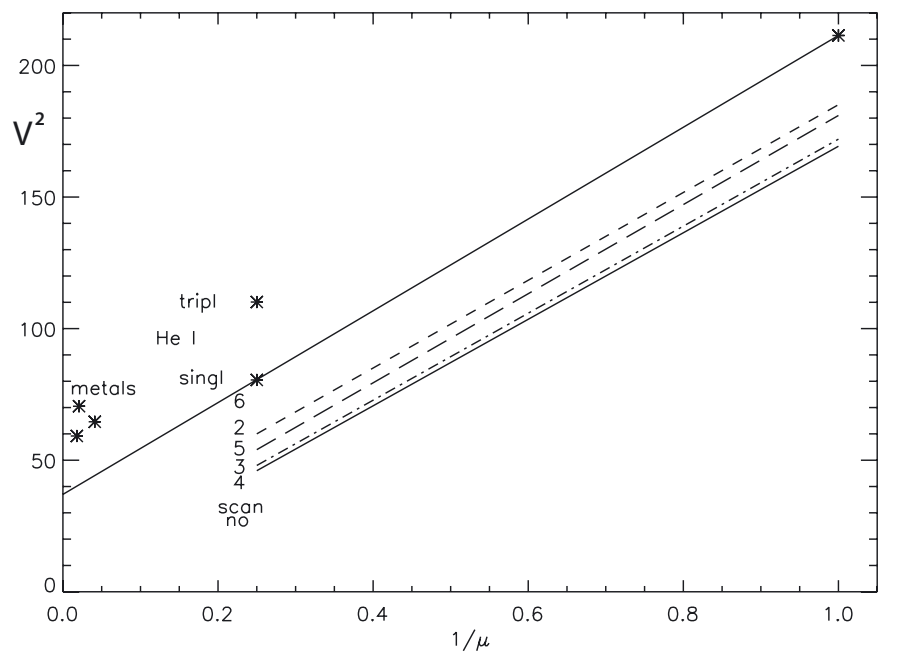

Fig. 8. Spatial variation of $V^{2}=\left(c \cdot \Delta \lambda_{\mathrm{D}} / \lambda_{0}\right)^{2}$ versus inverse atomic mass $1 / \mu$ for 5 spatially distinct scans in prominence No. 1 . Asterisks give scan-6 (in Fig. 7); the full line connecting $\mathrm{H} \gamma$ and He I 5015 (singlet), triplet and metals lay above that line (as in Fig. 1). The other lines similarly connect $\mathrm{H} \gamma$ and He 5015 (singlet) values observed for scans-2 through -5 (in Fig. 7), and are of nearly the same steepness, $T_{\text {kin }}=10650 \mathrm{~K}$; their ordinate off-sets correspond to $2 \leq V_{\text {nth }} \leq 6 \mathrm{~km} \mathrm{~s}^{-1}$.

of line broadening (along the slit; Fig. 7). If we plot a $1 / \mu$ diagram for each spatial location (scan-no.), we find nearly parallel curves (Fig. 8). Although their steepness of correspondingly $T_{\text {kin }}=10650 \mathrm{~K}$ is certainly unrealistic, their different ordinate shifts (corresponding to $2 \leq V_{\text {nth }} \leq 6 \mathrm{~km} \mathrm{~s}^{-1}$ ) indicate a spatial variation of non-thermal line broadening. This variation of physical states within prominences is also seen from the observed relations of $\mathrm{He}_{3}$ and $\mathrm{H} \beta$ (Stellmacher \& Wiehr 1995) showing characteristic mean ratios for individual prominences which, however, noticeably vary within the same prominence (Stellmacher et al. 2007).
A unique pair $\left[T_{\mathrm{kin}} ; V_{\mathrm{nth}}\right]$ cannot characterize a mean of prominence regions with individual physical state, averaged to assure a sufficiently high observational accuracy for the faint (metallic) lines. In our data we average over 3.4 arcsec (in the slit direction). If we also assume 3.4 arcsec for the influence of seeing during our (typically $10 \mathrm{~s}$ ) exposure, the effective spatial area contributing to the line profiles is $2500 \mathrm{~km} \times 2500 \mathrm{~km}$. If we assume that prominence structures have widths of $\leq 150 \mathrm{~km}$, separated by spaces of similar widths (see e.g. the speckle reconstructed $\mathrm{H} \alpha$ image in Wiehr et al. 2007; Fig. 8); our resolution area would contain at least 60 of such structures.

The number of threads along the line of sight may be estimated from calculations by GHV: their coolest model with $T_{\text {kin }}=4300 \mathrm{~K}$ does not reach our highest emission $\mathrm{E} \alpha=$ $4.4 \times 10^{5} \mathrm{erg} /\left(\mathrm{s} \mathrm{cm}^{2} \mathrm{ster}\right)$, observed in a massive prominence; their model with $T_{\text {kin }}=6000 \mathrm{~K}$ matches this emission assuming $D=8000 \mathrm{~km}$. Adopting this value, one obtains $\approx 27$ threads plus inter-threads (of totally $300 \mathrm{~km}$ ) along the line of sight, which fairly agrees with Gunár et al. (2012). The total prominence volume emitting our observed optically thin lines will then represent an integration over $27 \times 60 \approx 1600$ condensations.

Line-specific non-thermal broadening would thus indicate that the condensations have different physical states, since a temperature stratification (plus PCTR) within clumps of $\leq 150 \mathrm{~km}$, even when adding 27 of them along the line of sight, may hardly provide sufficient thickness to emit the observed radiance of $\mathrm{Na}$ I through He II to "hot" EUV lines (e.g. Stellmacher et al. 2003). Hence, each spectral line originates from a sub-entity of the $\approx 1600$ condensations, which exhibits appropriate conditions for its emission and causes a clump-specific non-thermal broadening. This is supported by the hierarchy of $V_{\text {nth }}$ for $\mathrm{H} \gamma$, $\mathrm{H} \delta, \mathrm{Mg} \mathrm{b}$ and $\mathrm{Mg} 4571$ (see end of Sect. 4.1). Threads of individual temperature and PCTR have already been proposed by Pojoga (1994), which differs from macro-shifted isothermal threads with equal PCTRs assumed by Gunár et al. (2008).

\subsection{Downdraft motions}

A scenario that predicts plasma clumps at various physical states has been modelled by Low et al. (2012). Their calculations show that condensations of cool gas must occur in prominences "as a consequence of the non-linear coupling of force balance and energy transport". The "catastrophic radiative cooling" causes the gas to become largely neutral, and the frozenin state breaks down. The clump then sinks and progressively "warms up by resistive heating" (Low et al. 2012) until, at sufficient re-ionization, the clump is eventually again frozen-in and stops sinking. This "recurrent break-down of the frozen-in condition" (Low et al. 2012) thus causes a relation between (sink) velocity and temperature.

If we reasonably assume that some fraction of the vertical falling motion shows up as non-thermal velocity (due to turbulence and/or shocks; cf. Hillier et al. 2012), a sub-entity of the 1600 clumps averaged in our spectra, which has the appropriate physical conditions for a specific line emission, causes a line-specific non-thermal broadening.

Downdraft motions in quiescent prominences have been observed with the HINODE instrument by Berger et al. (2010) from $\mathrm{H} \alpha$ and $\mathrm{Ca}$ II $\mathrm{H}$ images. These, however, primarily show the spatial variation of local brightenings rather than actual motions. We cannot a priori exclude that a change of their location reflects a spatial variation of locally optimal excitation conditions. Our line widths data, in turn, give strong indication for real plasma 
Table 2. Mean observed ratios of line broadening, $\Delta \lambda_{\mathrm{D}} / \lambda_{0}$, (upper) and radiance, $E_{\mathrm{tot}}$, (lower), valid for $0.8 \leq E_{\mathrm{tot}}(H \gamma) \leq 5.5 \times$ $10^{4} \mathrm{erg} /\left(\mathrm{cm}^{2} \mathrm{~s}\right.$ ster $)$.

\begin{tabular}{ccc}
\hline \hline & Emission lines & Ratios \\
\hline \multirow{3}{*}{$\Delta \lambda_{\mathrm{D}} / \lambda_{0}$} & $\mathrm{H} \delta / \mathrm{H} \gamma$ & $1.05 \pm 0.03$ \\
& $\mathrm{He}$ 4472 (tripl)/He I 5015 (singl) & $1.1 \pm 0.05$ \\
& $\mathrm{Mg} \mathrm{b}_{2}$ 5172/Mg I 4472 (tripl)) & $1.45 \pm 0.3$ \\
& $\mathrm{He} \mathrm{I} \mathrm{4472} \mathrm{(tripl)/He} \mathrm{I} \mathrm{5015} \mathrm{(singl)}$ & $1.3 \pm 0.3$ \\
\hline \multirow{3}{*}{$E_{\text {tot }}$} & $\mathrm{He} \mathrm{I} \mathrm{4472} \mathrm{(tripl)/He} \mathrm{II} \mathrm{4686}$ & $9.4 \pm 2$ \\
& $\mathrm{Mg} \mathrm{b}_{2}$ 5172/Mg 4571 & $35 \pm 4$ \\
\hline
\end{tabular}

velocities and can then be considered as an additional support for flows in quiescent prominences.

\subsection{Future modelling}

Prominence models may then consider a variety of clumps (of e.g. $150 \mathrm{~km}$ diameter) each with individual temperature and nonthermal broadening and sum them up along the line of sight (as e.g. done by Gunár et al. 2008) and over an area corresponding to the spatial resolution achieved in spectra (e.g. our $2500 \mathrm{~km} \times$ $2500 \mathrm{~km}$ ). The observed mean ratios of widths and radiance, listed in Table 2, may be used to scale these models.

These ratios do not markedly vary through a prominence (here along the slit) although the absolute values do (cf. Fig. 7). We note that the ratios are valid for an $\mathrm{H} \gamma$ radiance range of $0.8-5.5 \times 10^{4} \mathrm{erg} /\left(\mathrm{s} \mathrm{cm}^{2}\right.$ ster $)$, for which the GHV models give $\tau_{\text {alpha }}=2-20$. It is still unknown how these ratios will behave in faint prominences with pronounced structuring and $\tau_{\text {alpha }}<2$.

Acknowledgements. We are very indebted to F. Hessman for fruitful discussions; C. Le Men contributed valuable support with the THEMIS and M. Bianda and R. Ramelli with the IRSOL observations. We thank an unknown referee for helpful suggestions. J. Hirzberger (MPS) kindly put his reduction software to our disposal. SOLARNET provided financial support.

\section{References}

Berger, T., Slater, G., Hurlburt, N., et al. 2010, ApJ, 716, 1288 Gouttebroze, P., Heinzel, P., \& Vial, J.-C. 1993, A\&AS, 99, 513

Gunár, S., Heinzel, P., Anzer, U., \& Schmieder, B. 2008, A\&A, 490, 307

Gunár, S., Mein, P., Schmieder, B., Heinzel, P., \& Mein, U. 2012, A\&A, 543, A93

Hillier, A., Isobe, H., Shibata, K., \& Berger, T. 2012, ApJ, 756, 110

Jejcic, S., Heinzel, P., Zapiór, M., et al. 2014, Sol. Phys., 289, 2847

Landman, D. A. 1981, ApJ, 251, 768

Landman, D. A. 1985, ApJ, 295, 220

Low, B. C., Liu, W., Berger, T., \& Casini, R. 2012, ApJ, 757, L21

Park, H., Chae, J., Song, D., et al. 2013, Sol. Phys., 288, 105

Pojoga, S. 1994, in Solar Coronal Structures, eds. V. Rusin, P. Heizel, \& J. C. Vial, Proc. IAU Coll., 144, 357

Ramelli, R., Stellmacher, G., Wiehr, E., \& Bianda, M. 2012, Sol. Phys., 281, 697 Sotirovski, P. 1965, Ann. d'Astrophys., 28, 1065

Stellmacher, G., \& Wiehr, E. 1970, A\&A, 7, 432

Stellmacher, G., \& Wiehr, E. 1994, A\&A, 290, 655

Stellmacher, G., \& Wiehr, E. 1995, A\&A, 299, 921

Stellmacher, G., \& Wiehr, E. 2000, Sol. Phys., 196, 357

Stellmacher, G., \& Wiehr, E. 2005, A\&A, 431, 1069

Stellmacher, G., Wiehr, E., \& Dammasch, I. E. 2003, Sol. Phys., 217, 133

Stellmacher, G., Wiehr, E., \& Hirzberger, J. 2007, Sol. Phys., 240, 25

Wiehr, E., \& Stellmacher, G. 2015, Cent. Eur. Astrophys. Bull., in press

Wiehr, E., Stellmacher, G., Ramelli, R., \& Bianda, M. 2013, Cent. Eur. Astrophys. Bull., 37, 487 